\title{
Image Inpainting Techniques Combined with Isolated Pixel Filtering Applied to Multifunctional Drawing Robots
}

\author{
Chun-Chieh Wang ${ }^{1}$, Zhan-Xian Ye ${ }^{2}$ \\ ${ }^{I}$ Department of Automation Engineering and Institute of Mechatronoptic Systems, Chienkuo Technology University, Taiwan \\ (Tel: 886-4-7111111ext3350, Fax: 886-4-7111164) \\ 1jasonccw@ctu.edu.tw \\ ${ }^{2}$ Department of Electronic Engineering, Chienkuo Technology University, Taiwan
}

\begin{abstract}
The purpose of this thesis is to assist street painters to improve their work efficiency. That is, the simpler part of the drawing is drawn by the robot. Once the initial picture is completed, the artist will take over the painting. In this paper, HSI color space is used to improve the effect of color simplification such that the recognition of image processing results is enhanced. Moreover, the isolated pixel filtering is used to replace the less-affected isolated point color with the surrounding color. Furthermore, image inpainting techniques are utilized to reduce the distortion caused by the isolated pixel filtering. Besides, we adjusted the path planning as well as reduced isolated points to dramatically reduce drawing time. At the same time, LabVIEW can issue commands directly to control the robot by adding the communication function. In addition, to achieve the multifunctional drawing robotic mode, this system adds the sketch function. Through the image resolution adjustment as well as the shortening of the spacing of the drawing lines, the robot can draw more detailed pictures in the same size of drawing space. The measured results confirm that the application of the technology in this paper can shorten the drawing time by about $55 \%$ on the multifunctional drawing robot system.

Keywords: Isolated pixel filtering, Image inpainting, Watercolor, Sketch, Multifunctional drawing robots
\end{abstract}

\section{INTRODUCTION}

In view of that many street painters usually takes a long time to draw a picture such that it is impossible to produce a large amount in a short time. Therefore, we hope to develop a drawing robot that can assist painters. With the help of robots, the artist can complete more paintings in the same time and improve drawing efficiency.

In the literature of robot drawing in the past, the image detection applied in a watercolor painting robot proposed by Hung YY [1] in 2017. It is characterized by using HSV color simplification method with a self-made drawing mechanism, and then using a limited variety of colors to complete the drawing. Although the recognition of the image is acceptable, the long drawing time is a major disadvantage. In 2016, Hong M.J. [2] proposed robotics artistic colorful picture drawing and painting using visual feedback control system. Two different painting styles were used. 2017 Columbia University Creative Machines Lab / PIX18 [3] uses Gantry multi-axis robotic arm to draw pictures with artificial intelligence. In the same year, Massachusetts Institute of Technology Dane Kouttron et al.
[4] used online PNG to SVG software to simplify the colors used Linux $\mathrm{CNC}$ with a multi-axis robotic arm for drawing.

For the sketch drawing part, Lin L.C. [5] used edge detection to generate contour images and used mobile robots to draw on a flat surface in 2017. In 2009, Mac T.T. [6] and Chuang L.W. [7] used PI and PID controllers to adjust motors to control multi-axis robotic arms to draw portraits, respectively. The above three documents are all drawn with the edge of the image. In 2016, Jhang G.M. et al. [8] used multi-layer binary images to overlap drawing. It focused on the performance of shadows to achieve the effect of light and shadow drawing.

In order to improve the above shortcomings, we apply image inpainting techniques combined with isolated pixel filtering to multifunctional drawing robots.

\section{RESEARCH METHOD}

\subsection{HSI color model and color simplification}

Digital images often use the RGB color space to define colors. The parameters of the RGB color space are all connected, which makes image processing difficult. In contrast, the parameters of the HSI color space are 
independent of each other, namely Hue $(\mathrm{H})$, Saturation $(\mathrm{S})$, Intensity (I). Although the HSI color model is very similar to the HSV color model, there is a difference in the representation of the Intensity of HSI and the Value of HSV, as shown in Fig.1. Fig. 1(a) is a chromatogram image created by using the S and V parameters in HSV. Fig. 1(b) is a chromatographic image created by using the $\mathrm{S}$ and I parameters in HSI. From these two color spectrums, it can be found that the overall color change of the HSI color model is relatively smooth. In contrast, the HSV color model has more compact changes between light colors and primary colors, and it is more difficult to set the color reduction range. Therefore, this article uses the HSI color model with smoother color changes for color simplification. The advantage is that the parameter range setting is more flexible.

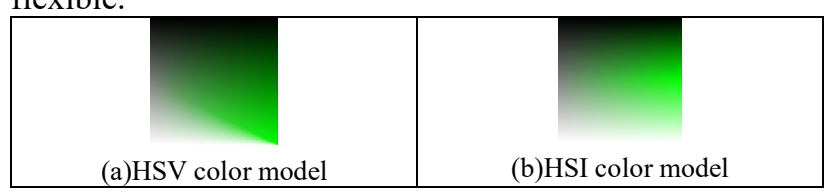

Fig.1. Comparison of HSV and HSI Green Chromatogram

In this paper, the scanning method from left to right and top to bottom is used to perform HSI color simplification on the color of each pixel. First, the RGB value of the pixel color is captured through the system. Since the color reduction method uses the HSI color space for processing, it is necessary to convert the RGB value to the HSI value before further processing, as shown in Fig.2. Since the hue representation in HSV and HSI color models is the same, the hue part continues the setting of reference [1], and its hue color distribution is shown in Fig. 3.

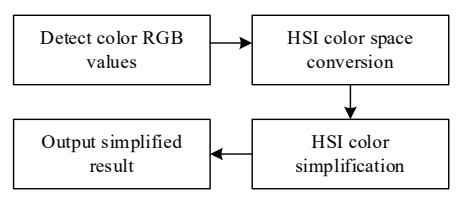

Fig. 2. The process of color simplification



After determining the range of hue, the range of 6 colors
(Dark, light, primary, black, gray and white) is set. Moreover, the color spectrum established by the HSI color space is used for adjustment. Since everyone's perception of color is different, this article is based on the author's perception of color to simplify the range of adjustments.

$I_{1}=128-S$

$I_{2}=\left|\frac{24448-(64 \times S)}{127}\right|$

$I_{3}=\left|\frac{8064-(64 \times S)}{127}\right|$

$I_{4}=128+S$

In Fig. 4, in the diagonal part, we use Eq.1 Eq4 to import the $\mathrm{S}$ value to get the relative range of I value. The scope of HSI color simplification is summarized in a rule table. Through the H, S, and I parameters in the captured target pixel, we can determine from the rule table which shade of color the pixel belongs to. In order to avoid errors in recognizing pictures with the naked eye due to too close shades, the 39 simplified colors are fine-tuned. The color codes used are shown in reference [9].

\subsection{Isolated pixel filtering for watercolor system}

In the process of image thumbnailing, the intersection of different colors is prone to color mixing. This change in color mixing is usually not too large. We want to remove the mixed color as much as possible and replace it with the surrounding colors. In addition, filtering out isolated pixels with low influence in the image can reduce the time it takes to repeatedly raise and lower the pen. Therefore, this paper proposes to filter out the isolated pixels and replace them with surrounding colors to eliminate the isolated pixels, so as to reduce the time consumed for lifting and lowering a single pixel. The method of filtering out isolated pixels starts from the upper left corner of the picture. A $3 \times 3$ matrix is used to scan from left to right and top to bottom. The center color of the matrix is used as the target for comparison. When the neighboring points have the same color, the original color of the target is maintained. If there is no same color, it is judged as an isolated pixel and replaced with the color with the largest surrounding area. As shown in Fig. 5(a), the central color is an isolated pixel, which will be replaced with the color of the largest area around it. When the surrounding eight pixels have the same color, they are not isolated points, as shown in Fig. 5 (b).

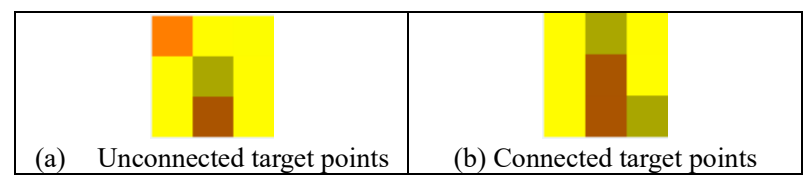

Fig. 5. Judgment of target point connectivity for watercolor (C) The 2021 International Conference on Artificial Life and Robotics (ICAROB2021), January 21 to 24, 2021 
When there are two or more colors with the largest pixel area around the target, the system will scan from left to right and then from top to bottom according to the scanning order, starting from the top left pixel, as shown in Fig. 6. After that, the first and second ranking colors are taken out. The HSI color space is used to convert these two colors to the target color. At the same time, $\mathrm{H}$ is used to determine which color is closer to the target color. After that, the color with a closer hue replaces the target color.

$$
\begin{array}{|l|l|l|}
\hline \text { (1) } & \text { (2) } & \text { (3) } \\
\hline \text { (4) } & \text { Target } & \text { (5) } \\
\hline \text { (6) } & \text { (7) } & \text { (8) } \\
\hline
\end{array}
$$

Fig. 6. Scan order of colors around the target

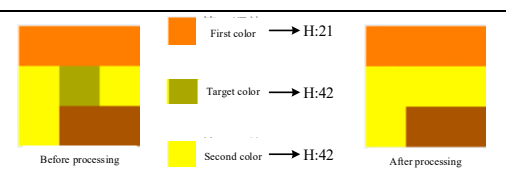

Fig. 7. Judgment method of the two largest color pixel areas around the target

Taking Fig. 7 as an example, since the number of orange and yellow pixels is 3 , it is impossible to determine which color replaces the target color. Therefore, it is necessary to judge by hue. First, it is determined by the scanning order that orange is the first color and yellow is the second color. Secondly, convert the color to HSI color space, and compare the $\mathrm{H}$ parameters with each other. The hue of the target color in the figure is the closest to the hue of the second-ranked color, so the target color is replaced by yellow. Fig. 8 is a comparison diagram before and after the isolated point filtering process.

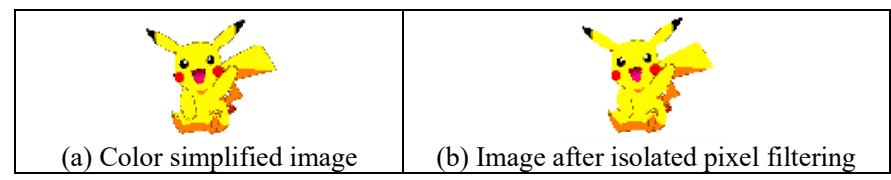

Fig. 8. Before and after filtering isolated pixels

\subsection{Feature inpainting for watercolor system}

After the isolated points are filtered out, some features in the image may be affected and cause distortion. The righthand line features are distorted, as shown in Fig. 8(b). Therefore, a new feature inpainting method is proposed in this article. That is, a $3 \times 3$ array is used to determine whether it needs to be inpaintinged. First, the images before and after the isolated points are filtered out are subtracted from each other. In the subtraction result, the part where the pixel value is not equal to 0 is the position filtered by the isolated point. The color of the pixels before the filtering of the processed position points is captured from the image before the isolated points are filtered. At the same time, in the image after the isolated points are filtered, these location pixels are found and centered on it. Then the $9 \times 9$ matrix around it is extracted. If the color of the remaining 8 cells except the center point in the matrix is the same as the color of the pixel before filtering, it is judged to be inpaintinged. This inpaintinging action is to restore the "pixel in the image after filtering out the isolated point" to the "color of the pixel before filtering out".

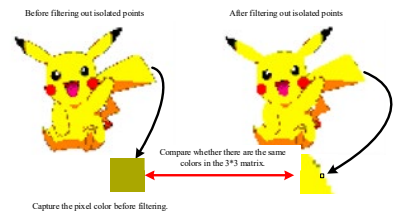

Fig. 9. Judgment method of image inpainting



Fig. 10. Comparison before and after image inpainting

\subsection{Optimized path movement for watercolor system}

The area of the watercolor painting system consists of three parts: drawing, paint dipping, and brush cleaning. When moving in the drawing area, you only need to move the pen up and down by $4 \mathrm{~mm}$. In the paint picking and brush cleaning area, the brush needs to be raised to $16 \mathrm{~mm}$. Adjust the pen lift height for different areas to improve efficiency. Fig. 11 is a schematic diagram of the movement of the brush.

\subsection{Grayscale}

Most common images are color images in RGB color space. However, the common sketches are painted in black, white and gray. Therefore, we convert the RGB image into a grayscale image. The conversion formula is as follows: Gray $=0.299 R+0.587 G+0.114 B$

\subsection{Binarization}

We use binarization to divide the grayscale image into black and white. Black represents the area not to be drawn, and white represents the area to be drawn. By setting 4 different thresholds, the specific part of the grayscale image is captured, as shown in Fig. 12. 




Fig. 11. Schematic diagram of the movement of the brush



Fig. 12. Results of multiple binarization: (a) grayscale; binarization-(b) the shallowest (c) the second shallow (d) the second deep (e) the deepest

\subsection{Isolated pixel filtering and image inpainting for sketch system}

The isolated point filtering and image inpainting in the sketch system will process the black and white pixels in the binary image, as shown in Fig. 13. If there is the same color around the target point, it is judged as connected. Conversely, if there is no same color around the target point, it is judged as an isolated point. The image inpainting method is the same as in section 2.2

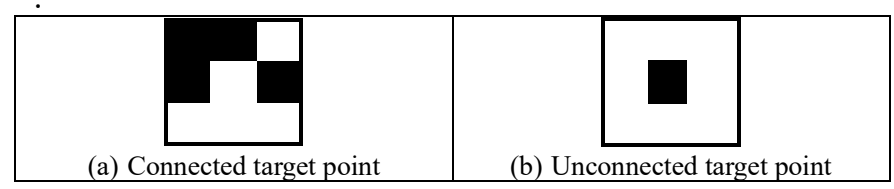

Fig. 13. Judgment of target point connectivity for sketch

\subsection{Drawing method for sketch}

We use lines in different directions for multi-level depiction. Draw the four shades of images mentioned earlier with lines in different directions, as shown in Fig. 14 The more the lines are stacked, the darker the color. In this way, the changes in depth are expressed.

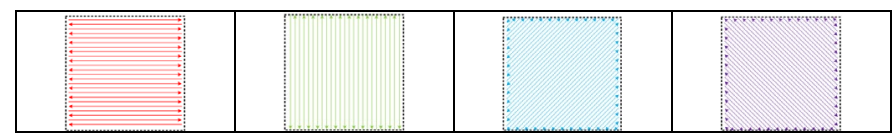

Fig. 14. Drawing trajectory in four directions

\section{SYSTEM ARCHITECTURE OF DRAWING ROB OTS}

\subsection{Watercolor system}

The process of watercolor drawing system is shown in Fig. 15. The direct connection between the drawing platform and the computer replaces the previous steps of manually loading data into the drawing platform to complete the automation of the watercolor painting system.

\subsection{Sketch system}

The process of the sketch system is shown in Fig.16. By adjusting the image resolution and the line spacing of the drawing track, the drawing results are more detailed.

\subsection{Communication system}

To improve the convenience of use, we have added a communication part to the program. The user can decide whether to draw directly with LabVIEW through the buttons in the HMI. After the program converts the processing result and saves it as a G-Code file, if the button in the human-machine interface is not pressed, the program will be directly terminated, allowing the user to transfer data through the SD Card. If the user presses the button, LabVIEW will connect with the robot and send the drawing path data for drawing.

\subsection{Configuration of drawing platform}

Fig. 17 (a) is the plane configuration of the drawing area. The physical drawing of the multifunctional drawing robot is shown in Fig. 17 (b).

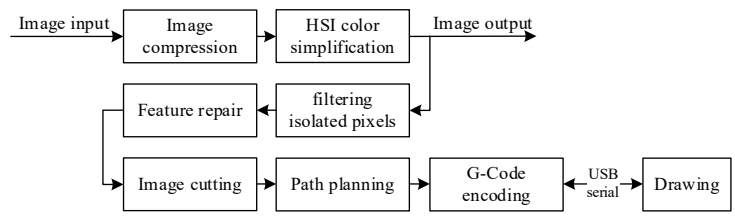

Fig. 15. The process of watercolor drawing system

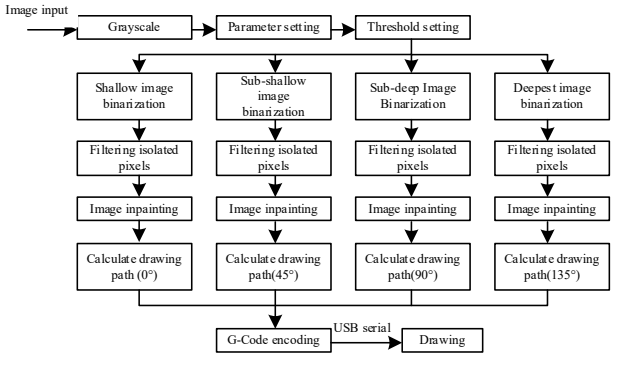

Fig. 16. The process of the sketch system 


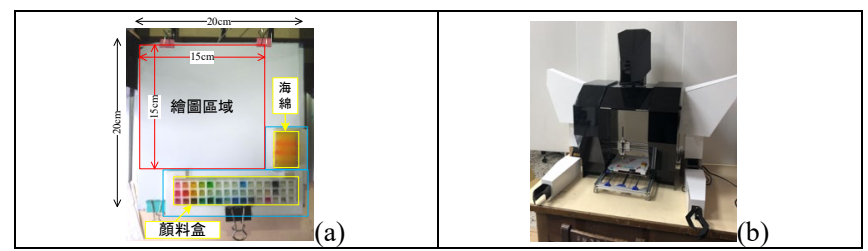

Fig. 17. (a) The plane configuration of the drawing area (b) The physical drawing of the robot

\section{EXPERIMENTAL RESULTS}

\subsection{Watercolor drawing results}

Fig. 18 shows the drawing results of Bill Gates photo.

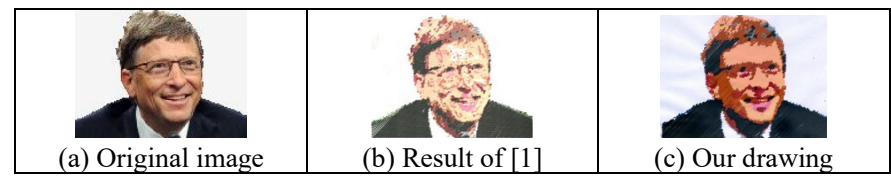

Fig. 18. The drawing results of Bill Gates photo

\subsection{Sketch drawing results}

Fig. 19 shows the drawing results of different line spacing. Although the drawing time with denser lines will increase a bit, the recognition of the portrait will be significantly improved.



Fig. 19. The drawing results of different line spacing

\section{CONCLUSION}

In this paper, HSI color space method strengthens the recognition of image processing results. Moreover, we use the isolated pixel filtering, image inpainting techniques and path planning optimization to effectively shorten the drawing time. In addition, through the image resolution adjustment as well as the shortening of the spacing of the drawing lines, the robot can draw more detailed pictures. Experimental results prove that image inpainting techniques combined with isolated pixel filtering have been successfully applied to multifunctional drawing robots.
Watercolor Painting Robot, Master Thesis of Chienkuo Technology University.

[2] Hong M.J. (2016), Robotics Artistic Colorful Picture Drawing and Painting Using Visual Feedback Control System, Master Thesis of National Taiwan University.

[3] Columbia University Creative Machines Lab PIX18 https://www.hodlipson.com/robotic-art.html (Search date:12/28/2017)

[4] Massachusetts Institute of Technology BABOT http://transistor-man.com/bluebot_revival.html (Search date: $12 / 28 / 2017)$

[5] Lin L.C. (2017), Trajectory Planning and Tracking Control of a FPGA-based Mobile Robot in a Picture Drawing Application, Master Thesis of National Taiwan University of Science and Technology.

[6] Mac T.T. (2009), Autonomous Drawing Manipulation of Humanoid Robot Arm, Master Thesis of National Taiwan University of Science and Technology.

[7] Chuang L.W. (2009), Human Portrait Generation System for Humanoid Robot Arm Drawing, Master Thesis of National Taiwan University of Science and Technology.

[8] Jhang G.M., Li C.J. (2016), Multilevel Based Image Recognition Applied in Automatic Sketch XYZ Platform, Graduation monograph of Chienkuo Technology University.

[9] Ye Z.X. (2018), Isolated Pixel Filtering and Image Inpainting Techniques Applied in System Improvement of Multifunctional Drawing Robots, Master Thesis of Chienkuo Technology University.

\section{REFERENCES}

[1] Hung Y.Y. (2017), Image Detection Applied in a

(C) The 2021 International Conference on Artificial Life and Robotics (ICAROB2021), January 21 to 24, 2021 